\title{
Late-Onset Inflammatory Bowel Disease-Like Syndrome after Ipilimumab Therapy: A Case Report
}

\author{
Reem Akel Bilal Anouti Arafat Tfayli \\ Division of Hematology and Oncology, Department of Internal Medicine, American \\ University of Beirut, Beirut, Lebanon
}

\section{Keywords}

Ipilimumab $\cdot$ Colitis $\cdot$ Inflammatory bowel disease $\cdot$ Melanoma $\cdot$ Immunotherapy $\cdot$ Side effects $\cdot$ Toxicity

\begin{abstract}
Background: Antitumor immunotherapy has become a major player in cancer therapy. Ipilimumab is a humanized monoclonal antibody against the cytotoxic $\mathrm{T}$ lymphocyte-associated antigen 4 (CTLA-4), an important downregulator of T-cell activation. Ipilimumab has demonstrated tumor regression and improvement in overall survival in patients with metastatic melanoma. Unfortunately, immune activation induced by this drug has been associated with several immune-mediated adverse effects, namely diarrhea and colitis. Case Presentation: We report the case of a 71-year-old male patient diagnosed with BRAF wild-type metastatic melanoma treated with three cycles of ipilimumab, after which he developed grade 3 enteritis. The patient improved on treatment with steroids, and ipilimumab was permanently discontinued at this point. Three years later, the patient's diarrhea returned and colonoscopy revealed active chronic colitis with ulceration resembling inflammatory bowel disease. He was started on Asacol (mesalamine). The patient did not report extraintestinal symptoms typically associated with inflammatory bowel disease, nor did he have a personal or family history of bowel disorders. Moreover, his presentation was not typical of inflammatory bowel disease in the elderly. Conclusion: Our findings suggest a link between ipilimumab-induced grade 3 enteritis and late-onset inflammatory bowel disease-like syndrome. To our knowledge, the case is the first in the literature to report late-onset inflammatory bowel disease-like syndrome years after discontinuation of ipilimumab treatment.




\section{Case Reports in Oncology}

\begin{tabular}{l|l}
\hline Case Rep Oncol 2017;10:456-461 \\
\hline DOI: 10.1159/000475709 & $\begin{array}{l}\text { C 2017 The Author(s). Published by S. Karger AG, Basel } \\
\text { www.karger.com/cro }\end{array}$ \\
\hline
\end{tabular}

Akel et al.: Late-Onset Inflammatory Bowel Disease-Like Syndrome after Ipilimumab Therapy: A Case Report

\section{Introduction}

Antitumor immunotherapy has become a major player in cancer therapy. Ipilimumab is a humanized monoclonal antibody against the cytotoxic $\mathrm{T}$ lymphocyte-associated antigen 4 (CTLA-4), an important downregulator of T-cell activation [1-3]. Ipilimumab has demonstrated tumor regression and improvement in overall survival in patients with metastatic melanoma [1]. Unfortunately, immune activation induced by this drug has been associated with several immune-mediated adverse effects, namely diarrhea and colitis [1-7].

We report the case of a male patient diagnosed with metastatic melanoma treated with three cycles of ipilimumab, after which he developed grade 3 enteritis. The patient improved on treatment with steroids. Three years later, the patient's diarrhea returned and colonoscopy revealed active chronic colitis with ulceration resembling inflammatory bowel disease (IBD). To our knowledge, the case is the first in the literature to report a flare-up years after discontinuation of ipilimumab treatment.

\section{Case Report}

A.H. is a 71-year-old male patient known to have hypertension, dyslipidemia, coronary artery disease, and congestive heart failure who initially presented to our center on October 2011 for workup of an abdominal aortic aneurysm. CT angiography of the abdomen revealed a suspicious nodule within the right lower lobe of the lung. Further imaging and pathology revealed BRAF wild-type metastatic melanoma.

Prior to initiation of treatment, PET-CT examination revealed right lung lower lobe neoplastic nodule $17 \mathrm{~mm}$ in diameter (SUV $=5.8$ ) with secondary active lymph nodes affecting the pre-trachea, right para-trachea, pre-carina, and right axilla. The patient was initially started on temozolamide for 3 cycles without notable side effects, until ipilimumab became available at our center. Starting in February 2012, the patient received his first two cycles of ipilimumab with no reported side effects.

After his third cycle in April 2012, the patient reported watery nonbloody diarrhea reaching up to 20 episodes/day associated with severe intermittent cramping abdominal pain. He was diagnosed with grade 3 enteritis requiring oral prednisone $1 \mathrm{mg} / \mathrm{kg}$ twice daily. Ipilimumab was permanently discontinued at this point. His diarrhea and abdominal pain significantly improved on steroids over the next few weeks, allowing a successful steroid taper and discontinuation.

In June 2012, follow-up PET-CT scan showed a decrease in size of the right lower lung lobe nodule ( $\mathrm{dmax}=7 \mathrm{~mm}$, SUV $=0.9$ ) as well as a decrease in size and metabolic activity of previously noted active lymph nodes. By December 2012, the patient had clinically improved and PET-CT scan revealed no evidence of active disease with complete resolution of the right lung nodule.

The patient continued to have regular follow-up with no treatment provided and was clinically asymptomatic and in remission. However, in May 2015, the patient began complaining of intermittent watery nonbloody diarrhea nonresponsive to antibiotics and standard medical management. For further assessment, he underwent colonoscopy in November 2015 (Fig. 1), which revealed hypertrophied anal papilla and multiple long shallow ulcers with an erythematous edematous mucosa extending up to the sigmoid. Patchy inflammatory changes were noted throughout the entire colon (loss of vascular pattern, edema, ulcers). 


\section{Case Reports in Oncology}

The terminal ileum was normal in appearance. This pattern was consistent with an inflammatory bowel-like syndrome.

Biopsy results further corroborated this finding. Several fragments of benign colorectal mucosa, with mild to moderate architectural distortion associated with moderate increase in lamina propria mononuclear cells were found on microscopic histopathologic examination. Furthermore, patchy cryptitis, crypt abscesses, and ulceration were noted (Fig. 2). Moreover, there was no dysplasia, cytomegalovirus inclusions, or granulomas. The overall impression was that of active chronic colitis with ulceration consistent with IBD.

He was started on Asacol (mesalamine). The patient is still on mesalamine and his diarrhea is under control and partially resolved.

\section{Discussion}

This is a case of a 71-year-old gentleman with metastatic melanoma who developed grade 3 enteritis and diarrhea upon treatment with ipilimumab, requiring drug discontinuation and treatment with steroids to achieve clinical remission. This initial presentation is a well-known and commonly encountered side effect of the immune modulator ipilimumab $[1-3,5,6]$.

However, our literature review shows no published reports of late-onset IBD-like syndrome years after discontinuation of ipilimumab treatment as observed in this patient.

Berman et al. [1] conducted a multicenter phase II trial on 115 patients with unresectable stage III/IV melanoma receiving ipilimumab to assess the underlying mechanism of ipilimumab-induced enterocolitis. Histologic assessment of bowel biopsies in patients who developed grade 2 or higher diarrhea revealed active colitis characterized by neutrophilic infiltration of the lamina propria [1]. In addition, neutrophilic cryptitis, crypt abscesses, as well as mucosal erosions were noted [1]. However, histologic features of chronicity typically observed in Crohn's disease or ulcerative colitis were not evident [1]. This lead to the conclusion that ipilimumab-induced IBD-like syndrome may be a separate entity from IBD observed in untreated patients.

Moreover, Marthey et al. [5] found similar findings of acute but not chronic inflammation on endoscopic biopsies in patients with anti-CTLA-4 enterocolitis. Inflammation was also noted on follow-up colonoscopy of some patients up to 6 months after the onset of enterocolitis, suggesting that anti-CTLA-4-induced enterocolitis may progress to chronic IBD [5].

Following a symptom-free period of several years, our patient developed recurrence of diarrhea and abdominal pain. On colonoscopy, findings resembled both ulcerative colitis and Crohn's disease. Whereas patchy distribution of inflammation and anal involvement are more frequent features of Crohn's disease, continuous mucosal involvement of the sigmoid with disease-free terminal ileum are more suggestive of ulcerative colitis. Furthermore, histology revealed several findings consistent with previous studies by Berman et al. [1] and Marthey et al. [5] such as cryptitis and crypt abscesses. In contrast, our patient had features of chronicity, evident on histopathologic examination, namely crypt architectural distortion and mononuclear cell infiltrates.

This patient did not report extraintestinal symptoms typically associated with IBD, nor did he have a personal or family history of bowel disorders. In addition, IBD is a condition typically diagnosed in the younger population with only $10-15 \%$ of IBD cases being diagnosed above the age of 60 and even fewer diagnosed after the age of 70 [8]. The presentation 


\section{Case Reports in Oncology}

in the elderly tends to be bloody, with minimal abdominal pain, and more severe compared to the younger population [8]. The above-described case does not fit the typical presentation of IBD in the elderly.

\section{Conclusion}

This patient's past medical history and histopathologic findings suggest a link between ipilimumab-induced grade 3 enteritis and the development of late-onset IBD-like syndrome.

\section{Statement of Ethics}

The material in the manuscript has been acquired according to modern ethical standards and written informed consent of the patient has been obtained.

\section{Disclosure Statement}

None of the authors have any conflict of interest to declare.

\section{Funding Sources}

No funding was provided for this case report.

\section{Author Contributions}

All authors contributed equally to the literature search, data collection (including figures), and manuscript writing.

\section{References}

1 Berman D, et al: Blockade of cytotoxic T-lymphocyte antigen-4 by ipilimumab results in dysregulation of gastrointestinal immunity in patients with advanced melanoma. Cancer Immun 2010;10:11

$\checkmark 2$ Dubeau MF, et al: Drug-induced inflammatory bowel disease and IBD-like conditions. Inflamm Bowel Dis 2013;19:445-456.

-3 Freeman HJ: Colitis associated with biological agents. World J Gastroenterol 2012;18:1871-1874.

-4 Goethals L, et al: 18F-FDG PET/CT imaging of an anti-CTLA-4 antibody-associated autoimmune pancolitis. Eur J Nucl Med Mol Imaging 2011;38:1390-1391.

-5 Marthey L, et al: Cancer immunotherapy with anti-CTLA-4 monoclonal antibodies induces an inflammatory bowel disease. J Crohns Colitis 2016;10:395-401.

6 Mesonero F, et al: Ipilimumab-induced colitis: a new challenge for gastroenterologists (in Spanish). Gastroenterol Hepatol 2016;39:233-238.

7 Nancey S, et al: Blockade of cytotoxic T-lymphocyte antigen-4 by ipilimumab is associated with a profound long-lasting depletion of Foxp3 + regulatory T cells: a mechanistic explanation for ipilimumab-induced severe enterocolitis? Inflamm Bowel Dis 2012;18:E1598-E1600.

8 Hussain SW, Pardi DS: Inflammatory bowel disease in the elderly. Drugs Aging 2010;27:617-624. 


\section{Case Reports in Oncology}

\begin{tabular}{l|l}
\hline Case Rep Oncol 2017;10:456-461 \\
\hline DOI: 10.1159/000475709 & $\begin{array}{l}\text { C } 2017 \text { The Author(s). Published by S. Karger AG, Basel } \\
\text { www.karger.com/cro }\end{array}$ \\
\hline
\end{tabular}

Akel et al.: Late-Onset Inflammatory Bowel Disease-Like Syndrome after Ipilimumab Therapy: A Case Report
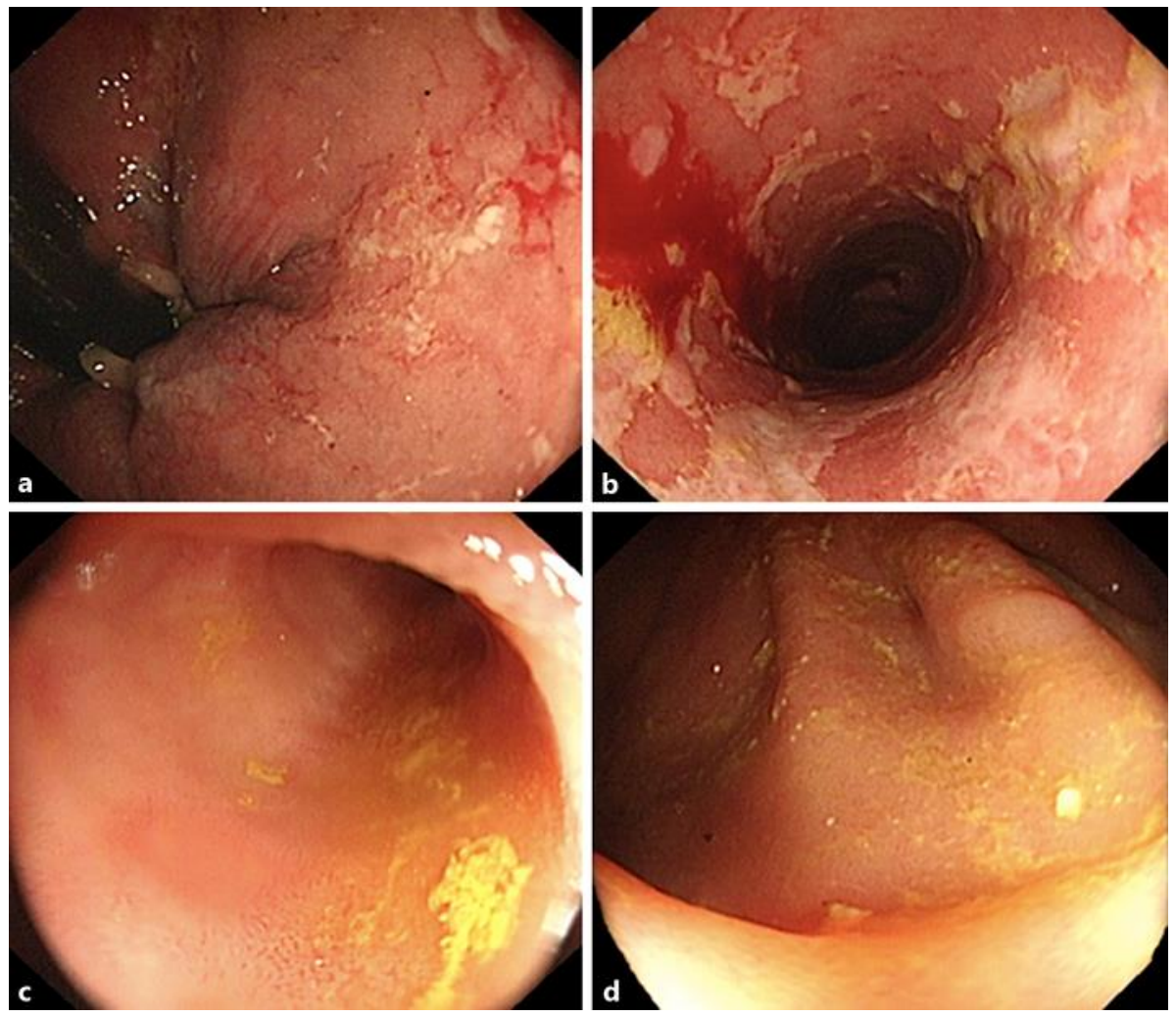

Fig. 1. Colonoscopy findings. a Hypertrophied anal papilla. b Multiple long shallow ulcers with an erythematous edematous mucosa extending up to the sigmoid. c Patchy inflammatory changes throughout the entire colon (loss of vascular pattern, edema, aphtous ulcers). d Normal terminal ileum. 


\section{Case Reports in Oncology}
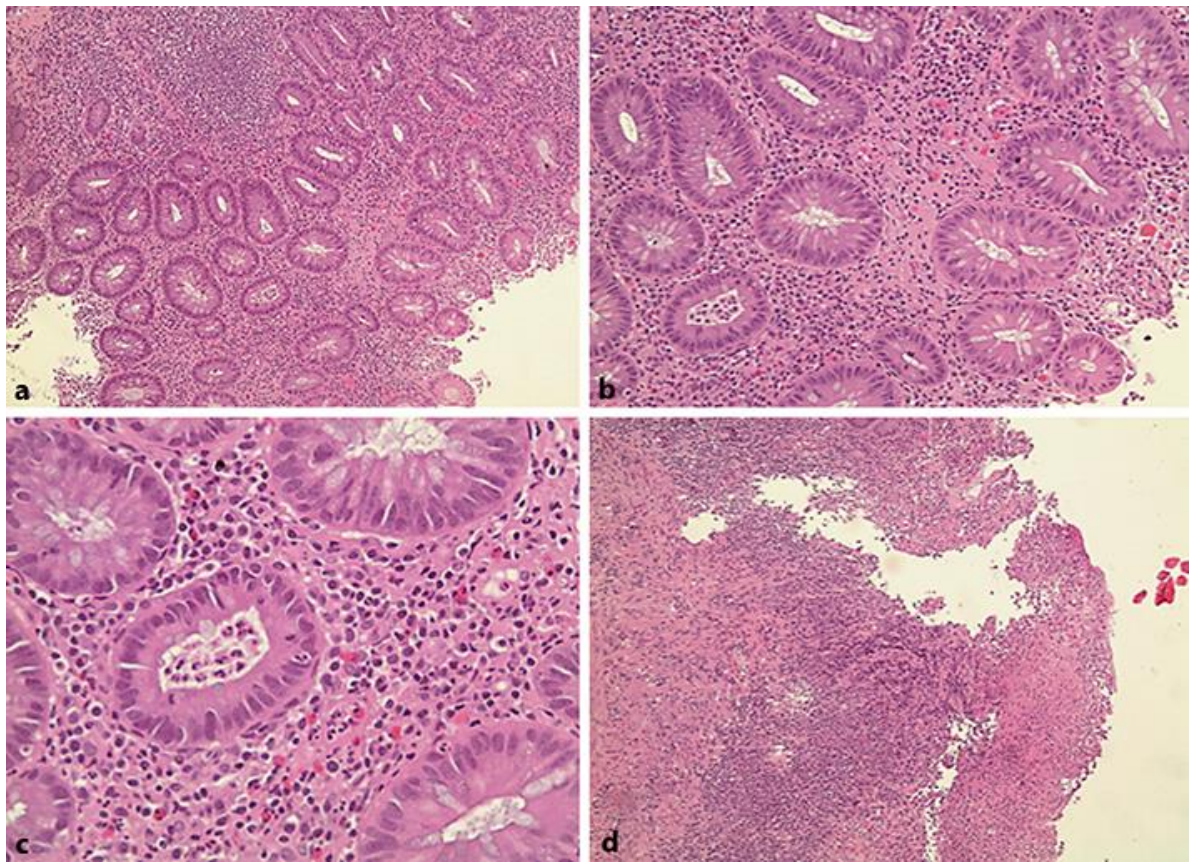

Fig. 2. Histopathologic examination of colonic biopsies. Representative hematoxylin and eosin section demonstrating active chronic colitis characterized by mild to moderate architectural distortion associated with moderate increase in lamina propria mononuclear cells (a), patchy cyptitis (b), crypt abscesses (c), and ulceration (d). 